\title{
HERMITIAN SYMMETRIC SPACES, CYCLE SPACES, AND THE BARLET-KOZIARZ INTERSECTION METHOD FOR CONSTRUCTION OF HOLOMORPHIC FUNCTIONS
}

\author{
Joseph A. Wolf
}

\begin{abstract}
Under certain conditions, a recent method of Barlet and Koziarz [2] constructs enough holomorphic functions to give a direct proof of the Stein condition for a cycle space. Here we verify those conditions for open $G_{0}$-orbits on $X$, where $G_{0}$ is the group of a bounded symmetric domain and $X$ is its compact dual viewed as a flag quotient manifold of the complexification $G$ of $G_{0}$. This Stein result was known for a few years [9], and in fact a somewhat more precise result is known [12] for the flag domains to which we apply the Barlet-Koziarz method, but the proof here is much more direct and holds the possibility of greater generality. Also, some of the tools developed here apply directly to open orbits that need not be measurable, avoiding separate arguments of reduction to the measurable case.
\end{abstract}

\section{Introduction}

Let $G_{0}$ be a real semisimple Lie group, $G$ its complexification, $Q$ a parabolic subgroup of $G$ and $X=G / Q$ the corresponding flag manifold, and $D$ an open $G_{0}$-orbit on $X$. Let $M_{D}$ denote the linear cycle space of $D$ (see (2.6) and (2.7) below). The usual proof [9] that $M_{D}$ is a Stein manifold, in the case where $D$ is measurable, i.e. where $D$ carries a $G_{0}$-invariant positive Radon measure, is rather indirect. One constructs a particular exhaustion function on $D$, uses it to verify that $D$ is $(s+1)$-complete where $s$ is the complex dimension of the cycles in $M_{D}$, and then in a rather technical way pushes the exhaustion function from $D$ to $M_{D}$. This is done in such a way that (a slight modification of) the resulting function on $M_{D}$ is a strictly plurisubharmonic exhaustion function there. If $D$ is not measurable, one studies [10] a minimal flag covering of $X$ and $D$.

Under certain conditions (2.11) the Barlet-Koziarz intersection method can show directly that $M_{D}$ is Stein. That is the new element of this paper. One uses the intersection method to construct enough holomorphic functions to show that $D$ is holomorphically convex, and at that point the Stein condition follows from some elementary conditions. The delicate points here are the $G_{0}$-orbit structure of the boundary of $D$ in $X$, construction of a certain transversal $X^{\prime}$ to the elements of $M_{D}$, and an implicit application of some results from intersection theory on the Chow ring of $X$. See Proposition 2.13.

Received February 14, 2000.

Research supported by the Alexander von Humboldt Foundation and by NSF Grant DMS 97 05709. The author thanks the Ruhr-Universität Bochum for hospitality. 
In Section 2 we describe the Barlet-Koziarz method and specialize it to flag domains.

In the case where $G_{0}=S U(p, q)$ and $X$ is the complex Grassmann manifold $\operatorname{Gr}(p, q)$, we verify the conditions (2.11) with classical computation. This is done in $\S 3$, and it is done in a way that indicates the procedure in a more general symmetric space setting. There are really two parts here: elucidation of the $G_{0}$-orbit structure of $X$ and construction of a certain sort of transversal to the cycles. The result is Proposition 3.12.

The general hermitian symmetric space considerations are in Sections 4 and 5. The $G_{0}$-orbit structure is described in $\S 4$ using partial Cayley transforms, which leads more or less directly to construction of the transversals in $\S 5$. The result is Proposition 5.17.

The conditions (2.11) were first used by Barlet and Huckleberry where $G_{0}=$ $S L(n+1 ; \mathbb{R})$ and $X$ is the complex projective space $P^{n}(\mathbb{C})$, so there is just one open orbit $D=P^{n}(\mathbb{C}) \backslash P^{n}(\mathbb{R})$. The cycles have codimension 1 in $D$ so one can use complex projective lines for transversals. The technique described here started when I noticed that their considerations, codimension 1 cycles and transversal projective lines, held as well for the case $G_{0}=S U(p, q)$ and $X=$ $P^{p+q-1}(\mathbb{C})$. Later, Huckleberry and Simon [3] carried out a complete analysis of the case where $G_{0}=S L(n+1 ; \mathbb{R})$ and $X$ is an arbitrary flag manifold of $G=S L(n+1 ; \mathbb{C})$.

I wish to thank Daniel Barlet and Alan Huckleberry for explaining their early example to me, Vincent Koziarz for his cooperation in keeping me informed as [2] progressed and for his comments on earlier versions of this paper, Robin Hartshorne for some discussions on intersection theory, and, especially, Alan Huckleberry for comments and suggestions on the organization and material in Section 2.

\section{The method}

Here is the method used for our examples, extracted from the more general results of [2] and reformulated for consistency with the usual notation of complex flag manifolds and hermitian symmetric spaces.

Let $D$ be an open submanifold of a complex projective variety $X$. Fix an integer $s \geqq 0$. Fix irreducible components $\mathcal{C}_{s}^{0}(D)$ and $\mathcal{C}_{s}^{0}(X)$ in the respective Barlet cycle spaces of (complex) dimension $s$ in $D$ and $X$, such that $\mathcal{C}_{s}^{0}(D) \subset$ $\mathcal{C}_{s}^{0}(X)$. Choose

$$
X^{\prime} \subset X \text { : closed nonsingular subvariety of } X \text { such that }
$$

(i) $\operatorname{codim}\left(X^{\prime} \subset X\right)=s$,

(ii) $X^{\prime}$ meets every element $Y \in \mathcal{C}_{s}^{0}(D)$, and

(iii) $X^{\prime \prime}=X^{\prime} \cap D$ is a Stein manifold. 
Let

$$
\begin{aligned}
& f: X^{\prime \prime} \rightarrow C \text { be a holomorphic function, and define } \\
& F: \mathcal{C}_{s}^{0}(D) \rightarrow \mathbb{C} \text { by } F(Y)=\sum_{y \in X^{\prime}(b) \cap Y} f(y) .
\end{aligned}
$$

Lemma 2.3. Suppose that (2.1) holds. Let $Y \in \mathcal{C}_{s}^{0}(D)$. Then $X^{\prime} \cap Y$ is finite. In particular $F$ is well defined in (2.2).

Proof. Hypothesis (2.1) says that $X^{\prime}$ meets $Y . X^{\prime} \cap Y \subset D$ because $Y \subset D$. $X^{\prime} \cap Y$ is the intersection of the compact variety $Y$ with the Stein manifold $X^{\prime \prime}=X^{\prime} \cap D$, so it is a compact subvariety of $X^{\prime \prime}$, and thus finite.

Now we can state a special case of [2, Proposition 1]), the basic step in the Barlet-Koziarz intersection method for construction of holomorphic functions on cycle spaces:

Proposition 2.4. If (2.1) and (2.2) hold, then $F$ is holomorphic on $\mathcal{C}_{s}^{0}(D)$.

Now that we have a construction of holomorphic functions, we look at holomorphic convexity. Here is a special case of [2, Proposition 3].

Proposition 2.5. For every $Y$ in the boundary bd $\left(\mathcal{C}_{s}^{0}(D)\right)$ of $\mathcal{C}_{s}^{0}(D)$ in $\mathcal{C}_{s}^{0}(X)$, suppose that there is a subvariety $X^{\prime} \subset X$ such that (i) $X^{\prime}$ satisfies (2.1) and (ii) $X^{\prime} \cap Y$ meets the boundary $\operatorname{bd}(D)$ of $D$ in $X$. Then $\mathcal{C}_{s}^{0}(D)$ is holomorphically convex.

Suppose in addition that if $Y_{1} \neq Y_{2}$ in $\mathcal{C}_{s}^{0}(D)$ then there is a closed subvariety $X^{\prime} \subset X$ such that (i) $X^{\prime}$ satisfies (2.1) and (ii) $X^{\prime} \cap Y_{1} \neq X^{\prime} \cap Y_{2}$. Then $\mathcal{C}_{s}^{0}(D)$ is Stein.

We reformulate Proposition 2.5 for the special case where $G_{0}$ is a real semisimple Lie group, $G$ is its complexification, $Q$ is a parabolic subgroup of $G, X$ is the complex flag manifold $G / Q$, and $D$ is an open $G_{0}$-orbit in $X$. We refer to this case as the "flag domain case."

In the flag domain case, we have a particular cycle

$$
Y_{0}=K_{0}\left(x_{0}\right)=K\left(x_{0}\right)
$$

where $D=G_{0}\left(x_{0}\right), K_{0}$ is an appropriately chosen maximal compact subgroup of $G_{0}$, and $K$ is the complexification of $K_{0}$. See [7] for the fact that $Y_{0}$ is a complex flag manifold sitting as a maximal compact subvariety of $D$. Here $s=\operatorname{dim}_{\mathbb{C}} Y_{0}$ and we make use of

$$
M_{D}:=\text { topological component of } Y_{0} \text { in }\left\{g Y_{0} \mid g \in G \text { and } g Y_{0} \subset D\right\} \text {. }
$$

rather than $\mathcal{C}_{s}^{0}(D)$. Observe that

$$
E:=\left\{g \in G \mid g Y_{0}=Y_{0}\right\} \text { is a closed complex subgroup of } G \text {. }
$$

Then $M_{D}$ has topology and complex structure as an open submanifold of

$$
M_{X}=\left\{g Y_{0} \mid g \in G\right\} \cong G / E \text {. }
$$


Here (2.9) is the definition of $M_{X}$. See [9] or [12] for details.

We may assume that $X$ is irreducible, i.e. that $G$ is simple. For everything breaks up as a product according to the decomposition of $G$ as a product of simple closed normal subgroups. If $G_{0} / K_{0}$ is not a bounded symmetric domain then the Lie algebra $\mathfrak{e}$ of $E$ is a maximal subalgebra of the Lie algebra $\mathfrak{g}$ of $G$, so either $E=G$ with $G_{0}$ transitive on $X$, or $K$ is the identity component $E^{0}$ of $E$. In these cases $M_{X}$ is an affine variety. If $G_{0} / K_{0}$ is a bounded symmetric domain then there is another possibiity, $E=K M^{ \pm}$, parabolic subgroup of $G$ such that $G / K M^{ \pm}$is the hermitian symmetric flag manifold dual to $G_{0} / K_{0}$. In this case $M_{X}$ is a projective variety.

$\mathcal{C}_{s}^{0}(D)$ denotes the irreducible component of the Barlet cycle space of $D$ that contains $M_{D}$, and $\mathcal{C}_{s}^{0}(X)$ is the irreducible component of the Barlet cycle space of $X$ that contains $M_{X}$.

Lemma 2.10. The inclusions $M_{D} \hookrightarrow \mathcal{C}_{s}^{0}(D)$ and $M_{X} \hookrightarrow \mathcal{C}_{s}^{0}(X)$ are holomorphic. If (2.1) and (2.2) hold, then $F: M_{D} \rightarrow \mathbb{C}$ is holomorphic.

Proof. The complex structure on $\mathcal{C}_{s}^{0}(X)$ is $G$-invariant, so each $G$-orbit on $\mathcal{C}_{s}^{0}(X)$ is a complex submanifold, and in particular $M_{X}$ is a complex submanifold of $\mathcal{C}_{s}^{0}(X)$. Now the open submanifold $M_{D}$ of $M_{X}$ is a complex submanifold of $\mathcal{C}_{s}^{0}(X)$ contained in $\mathcal{C}_{s}^{0}(D)$, thus is a complex submanifold of $\mathcal{C}_{s}^{0}(D)$. Thus the inclusions are holomorphic and the last statement is an immediate consequence of Proposition 2.4.

It is not quite as easy to carry Proposition 2.5 over from $\mathcal{C}_{s}^{0}(D)$ to $M_{D}$, but one can suitably adjust the statement and proof for the flag domain case. There (2.1) plus the hypothesis of the first part of Proposition 2.5 will be replaced by the condition:

There is a closed complex submanifold $X^{\prime} \subset X$ such that

(i) $\operatorname{codim}\left(X^{\prime} \subset X\right)=s$,

(ii) $x_{0} \in X^{\prime} \cap Y_{0}$ and $X^{\prime}$ is transversal to $Y_{0}$ at $x_{0}$,

(iii) $X^{\prime \prime}=X^{\prime} \cap D$ is a Stein manifold, and

(iv) $X^{\prime}$ meets every $G_{0}$-orbit on $\operatorname{bd}(D)$.

The hypothesis of the second part of Proposition 2.5 will not be an issue.

A standard and straightforward intersection number argument gives

Lemma 2.12. In the flag domain case, let $X^{\prime}$ be a closed complex submanifold of $X$ such that (i) $\operatorname{codim}\left(X^{\prime} \subset X\right)=s$, (ii) $X^{\prime}$ meets the base cycle $Y_{0}$, and $X^{\prime}$ is transversal to $Y_{0}$ in at least one intersection point, and (iii) if $Y \in M_{D}$ then $X^{\prime} \cap Y$ is finite. Then $X^{\prime} \cap Y$ is non-empty for every $Y \in M_{D}$.

Proposition 2.13. In the flag domain case, if (2.11) holds, then $M_{D}$ is Stein. 
Proof. Write $\operatorname{cl}(\cdot)$ and $\mathrm{bd}(\cdot)$ for Zariski closure and for boundary in the compact variety $\mathcal{C}_{s}^{0}(X)$. Then $M_{X}$ is Zariski-open in $\operatorname{cl}\left(M_{X}\right)$ because the action of $G$ on $\mathcal{C}_{s}^{0}(X)$ is algebraic. Now $\operatorname{cl}\left(M_{X}\right)$ is a compact subvariety of $\mathcal{C}_{s}^{0}(X)$, and $\operatorname{cl}\left(M_{X}\right)$ is a disjoint union $M_{X} \cup F$ where $F \subset \operatorname{bd}\left(M_{X}\right)$ is a union of lowerdimensional subvarieties. Now $\operatorname{bd}\left(M_{D}\right)$ is a disjoint union $\left(\operatorname{bd}\left(M_{D}\right) \cap F\right) \cup B$. Here $B \subset \operatorname{bd}\left(\mathcal{C}_{s}^{0}(D)\right)$ because $B \subset M_{X}$.

Let $\left\{Y_{n}\right\} \subset M_{D}$ with $\left\{Y_{n}\right\} \rightarrow Y \in \operatorname{bd}\left(M_{D}\right)$. Now either $Y \in B$ or $Y \in$ $\operatorname{bd}\left(M_{X}\right)$.

Suppose $Y \in B . \mathcal{C}_{s}^{0}(D)$ is holomorphically convex by Proposition 2.5 and Lemma 2.12. Thus we have $F: \mathcal{C}_{s}^{0}(D) \rightarrow \mathbb{C}$ holomorphic with $\lim \left|F\left(Y_{n}\right)\right|=\infty$.

Suppose $Y \in \operatorname{bd}\left(M_{X}\right)$. Then $\operatorname{bd}\left(M_{X}\right)$ is not empty, so $M_{X}$ cannot be projective. Now $M_{X}$ is affine, thus Stein, so we have $F: M_{X} \rightarrow \mathbb{C}$ holomorphic with $\lim \left|F\left(Y_{n}\right)\right|=\infty$.

Now $M_{D}$ is holomorphically convex.

If $Y_{1} \neq Y_{2}$ in $\mathcal{C}_{s}^{0}(D)$ and $x \in Y_{1} \cap Y_{2} \cap X^{\prime}$ then we have $g \in G_{0}$ with $g(x) \in Y_{1} \backslash Y_{2}$, and we replace $X^{\prime}$ by $g\left(X^{\prime}\right)$. So, as in the second part of Proposition 2.5, $M_{D}$ is Stein.

\section{Grassmann manifold example}

In this Section we work out the case where $X$ is the complex Grassmann manifold $\operatorname{Gr}(p, q)$ of $q$-dimensional linear subspaces of $\mathbb{C}^{p+q}$, and $G_{0}$ is an indefinite special unitary group $S U(p, q)$. This illustrates the situation where $X$ is an hermitian symmetric flag manifold, compact dual to a bounded symmetric domain $G_{0} / K_{0}$, treated in Sections 4 and 5 below. Of course one can skip this Section and go directly to $\S 4$ and $\S 5$.

Notation. If $\left\{u_{1}, \ldots, u_{\ell}\right\}$ are linearly independent vectors in $\mathbb{C}^{p+q}$ then $\left[u_{1} \wedge\right.$ $\left.\cdots \wedge u_{\ell}\right]$ denotes their span. Fix a basis $\left\{e_{1}, \ldots, e_{p+q}\right\}$ of $\mathbb{C}^{p+q}$ in which the hermitian form defining $G_{0}=S U(p, q)$ is given by $h(u, v)=\left(\sum_{1 \leqq i \leqq p} u_{i} \overline{v_{i}}\right)-$ $\left(\sum_{1 \leqq i \leqq q} u_{p+i} \overline{v_{p+i}}\right)$. Here $u=\sum u_{i} e_{i}$ and $v=\sum v_{i} e_{i}$. If $W$ is a subspace of $\mathbb{C}^{p+q}$ and $0 \leqq s \leqq \operatorname{dim} W$ then $G r(s, W)$ denotes the Grassmann manifold of $s$-dimensional subspaces of $W$. So $G r(s, W) \cong G r(r, s) \cong G r\left(s, \mathbb{C}^{r+s}\right)$ where $\operatorname{dim} W=r+s$.

The $S U(p, q)$-orbits on $\operatorname{Gr}(p, q)$ are given by the restriction of $h$ to the elements of the orbit. So the orbits are the

$$
\begin{aligned}
D_{a, b, c} & =\{x \in G r(p, q) \mid x \text { has sign and rank }(+,-, 0)=(a, b, c)\} \\
& =S U(p, q)\left(\left[e_{1} \wedge \cdots \wedge e_{a} \wedge e_{p+1} \wedge \cdots \wedge e_{p+b} \wedge f_{1} \wedge \cdots \wedge f_{c}\right]\right) \\
& \text { where } f_{j}=e_{p+1-j}+e_{p+q+1-j}
\end{aligned}
$$

Here of course $a \leqq p, b \leqq q, a+b+c=q$, and $c \leqq \min (p, q)$. The boundary is given by degeneration of $h$ on the elements of the orbit. Thus an orbit

$$
D_{a^{\prime}, b^{\prime}, c^{\prime}} \text { is in the closure of } D_{a, b, c} \text { if and only if } a^{\prime} \leqq a \text { and } b^{\prime} \leqq b \text {. }
$$


In particular, the open orbits are the

$$
D_{a}=D_{a, q-a, 0}=S U(p, q)\left(\left[e_{1} \wedge \cdots \wedge e_{a} \wedge e_{p+1} \wedge \cdots \wedge e_{p+q-a}\right]\right)
$$

and their boundaries are the union of the orbits given by

$$
\operatorname{bd}\left(D_{a}\right)=\bigcup_{\substack{0 \leqq i \leqq a, 0 \leqq j \leqq q-a, 0<i+j \leqq \min (p, q)}} D_{a-i, q-a-j, i+j} .
$$

Note that the bounded symmetric domain $G_{0} / K_{0}$ here is $D_{0}$, and its boundary orbits are the $D_{0, q-j, j}$ for $1 \leqq q \leqq \min (p, q)$. The boundary orbit

$$
D_{0, q-\min (p, q), \min (p, q)}
$$

is the Bergman-Shilov boundary of $D_{0}$.

Fix $D=D_{a}$ as in (3.3). It will be convenient to denote

$$
\mathbb{C}^{p+q}=S^{+} \oplus S^{-} \oplus T^{+} \oplus T^{-},
$$

where $S^{+}=\left[e_{1} \wedge \cdots \wedge e_{a}\right]$ and $T^{+}=\left[e_{a+1} \wedge \cdots \wedge e_{p}\right]$ are positive definite, and $S^{-}=\left[e_{p+1} \wedge \cdots \wedge e_{p+q-a}\right]$ and $T^{-}=\left[e_{p+q-a+1} \wedge \cdots \wedge e_{p+q}\right]$ are negative definite. Then $D_{a}=S U(p, q)\left(S^{+} \oplus S^{-}\right)$. The "base cycle" in $D_{a}$ is

$$
\begin{aligned}
Y_{0} & =S(U(p) \times U(q))\left(\left[e_{1} \wedge \cdots \wedge e_{a} \wedge e_{p+1} \wedge \cdots \wedge e_{p+q-a}\right]\right) \\
& =G r\left(a,\left[e_{1} \wedge \cdots \wedge e_{p}\right]\right) \times G r\left(q-a,\left[e_{p+1} \wedge \cdots \wedge e_{p+q}\right]\right) .
\end{aligned}
$$

In particular $s=\operatorname{dim}_{\mathbb{C}} Y_{0}=a(p+q-2 a)$. If $g \in S L(p+q ; \mathbb{C})$ and $Y=g Y_{0} \subset D_{a}$, then

$$
\begin{aligned}
& U=g\left(S^{+} \oplus T^{+}\right) \gg 0, V=g\left(S^{-} \oplus T^{-}\right) \ll 0, \mathbb{C}^{p+q}=U \oplus V, \text { and } \\
& Y=Y_{U, V}=\left\{U^{\prime} \oplus V^{\prime} \mid U^{\prime} \subset U, \operatorname{dim} U^{\prime}=a, V^{\prime} \subset V, \operatorname{dim} V^{\prime}=q-a\right\} .
\end{aligned}
$$

Let $G_{1} \cong G L(2 a ; \mathbb{C})$ denote the general linear group on $S^{+} \oplus T^{-}$, let $G_{2} \cong$ $G L((p-a)+(q-a) ; \mathbb{C})$ denote the general linear group on $T^{+} \oplus S^{-}$, and write $S\left(G_{1} \times G_{2}\right)$ for the determinant 1 elements of the product as a subgroup of $S L(p+q ; \mathbb{C})$. Our transverse manifold will be

$$
\begin{aligned}
X^{\prime} & =X_{a}^{\prime}=S\left(G_{1} \times G_{2}\right)\left(S^{+} \oplus S^{-}\right) \\
& =G_{1}\left(S^{+}\right) \times G_{2}\left(S^{-}\right)=G r\left(a, S^{+} \oplus T^{-}\right) \times G r\left(q-a, T^{+} \oplus S^{-}\right) .
\end{aligned}
$$

Then $X_{a}^{\prime} \cap D_{a}$ consists of all $W^{+} \oplus W^{-}$where $W^{+}$is an (a-dimensional) maximal positive definite subspace of $S^{+} \oplus T^{-}$and $W^{-}$is a $((q-a)$-dimensional) maximal negative definite subspace of $T^{+} \oplus S^{-}$. In particular

Lemma 3.9. $X_{a}^{\prime} \cap D_{a}$ is a bounded symmetric domain, and thus is a Stein manifold.

The next lemma illustrates Lemma 2.12 in our Grassmann manifold setting.

Lemma 3.10. The manifold $X_{a}^{\prime}$ meets every cycle $Y \in M_{D}$. 
Proof. Express $Y=Y_{U, V}$ as in (3.7). If $v \in \mathbb{C}^{p+q}$ decompose $v=v_{s,+}+v_{t,+}+$ $v_{s,-}+v_{t,-}$ with $v_{s, \pm} \in S^{ \pm}$and $v_{t, \pm} \in T^{ \pm}$. Now $v \mapsto v_{s,+}+v_{t,+}$ has negative definite kernel, hence is one to one on $U$, so $U$ has a basis $\left\{u_{i}=e_{i}+u_{i, s,-}+u_{i, t,-} \mid\right.$ $1 \leqq i \leqq p\}$. Similarly $V$ has a basis $\left\{v_{j}=e_{p+j}+v_{j, s,+}+v_{j, t,+} \mid 1 \leqq j \leqq q\right\}$.

Some $g_{1} \in G_{1}$, with block form matrix $\left(\begin{array}{cc}I & 0 \\ * & I\end{array}\right)$ relative to $S^{+} \oplus T^{-}$, sends $u_{i}$ to $u_{i}^{\prime}=e_{i}+u_{i, s,-}$ for $1 \leqq i \leqq a$. Similarly some $g_{2} \in G_{2}$, with block form matrix $\left(\begin{array}{ll}I & 0 \\ * & I\end{array}\right)$ relative to $S^{-} \oplus T^{+}$, sends $v_{j}$ to $v_{j}^{\prime}=e_{p+j}+v_{j, s,+}$ for $1 \leqq j \leqq q-a$. Now we have $g=\left(g_{1}, g_{2}\right) \in G_{1} \times G_{2}$ such that $g^{-1}\left[u_{1} \wedge \cdots \wedge u_{a}\right]$ has basis $\left\{u_{i}^{\prime}=e_{i}+u_{i, s,-} \mid\right.$ $1 \leqq i \leqq a\}$ and $g^{-1}\left[v_{1} \wedge \cdots \wedge v_{q-a}\right\}$ has basis $\left\{v_{j}^{\prime}=e_{p+j}+v_{j, s,+} \mid 1 \leqq j \leqq q-a\right\}$.

If we add an appropriate linear combination of these $v_{j}^{\prime}$ to $u_{i}^{\prime}$ we kill off their $u_{i, s,-}$ summands, changing $u_{i}^{\prime}$ to $u_{i}^{\prime \prime}=e_{i}+u_{i, s,+}^{\prime \prime}$ for $1 \leqq i \leqq a$. However each $u_{i, s,+}^{\prime \prime}$ is in the span $S^{+}$of $\left\{e_{1}, \ldots, e_{a}\right\}$. Now $g^{-1}\left[u_{1} \wedge \cdots \wedge u_{a} \wedge v_{1} \wedge \cdots \wedge v_{q-a}\right]$ has basis $\left\{e_{i} \mid 1 \leqq i \leqq a\right\} \cup\left\{v_{j}^{\prime} \mid 1 \leqq j \leqq q-a\right\}$. If we add an appropriate linear combination of these $e_{i}$ to $v_{j}^{\prime}$ we kill off their $v_{j, s,+}^{\prime}$ summands, changing $v_{j}^{\prime}$ to $e_{p+j}$. Now $\left.g^{-1}\left[u_{1} \wedge \cdots \wedge u_{a} \wedge v_{1} \wedge \cdots \wedge v_{q-a}\right]\right\}$ has basis $\left\{e_{i} \mid 1 \leqq i \leqq a\right\} \cup\left\{e_{p+j} \mid\right.$ $1 \leqq j \leqq q-a\}$

We have proved that $g\left(S^{+} \oplus S^{-}\right)=\left[u_{1} \wedge \cdots \wedge u_{a} \wedge v_{1} \wedge \cdots \wedge v_{q-a}\right]$. Thus $g\left(S^{+} \oplus S^{-}\right) \in Y=Y_{U, V}$. But $g\left(S^{+} \oplus S^{-}\right) \in X_{a}^{\prime}$ because $g$ was constructed as an element of $G_{1} \times G_{2}$. So $X_{a}^{\prime}$ meets $Y$.

Lemma 3.11. $X_{a}^{\prime}$ satisfies (2.11)(i), (2.11)(iii) and (2.11)(iv) for the open orbit $D_{a}$.

Proof. It follows from the definition (3.8) that the codimension of $X^{\prime}$ in $G r(p, q)$ is $p q-\left(a^{2}+(p-a)(q-a)\right)=a(p+q-2 a)=s$.

Lemma 3.9 says that $X_{a}^{\prime} \cap D_{a}$ is Stein.

The linear space $S^{+} \otimes T^{-}$on which $G_{1}$ acts, has a subspace $R_{1}$ of rank and sign $(a-i, 0, i)$ given by $R_{1}=\left[e_{1} \wedge \ldots e_{a-i} \wedge f_{1}^{\prime \prime} \wedge \cdots \wedge f_{i}^{\prime \prime}\right]$ where $f_{k}^{\prime \prime}=$ $e_{a+1-k}+e_{p+q+1-k}$. The linear space $T^{+} \otimes S^{-}$on which $G_{2}$ acts, has a subspace $R_{2}$ of rank and sign $(0, q-a-j, j)$, given by $R_{2}=\left[e_{p+1} \wedge \ldots e_{p+q-a-j} \wedge f_{1}^{\prime} \wedge \cdots \wedge f_{j}^{\prime}\right]$ where $f_{k}^{\prime}=e_{p+1-k}+e_{p+q-a+1-k}$. Thus $X_{a}^{\prime}$ contains the space $R_{1} \oplus R_{2}$, which has rank and sign $(a-i, q-a-j, i+j)$. In other words, $X_{a}^{\prime}$ meets the orbit $D_{a-i, q-a-j, i+j}$. In view of (3.4) now $X_{a}^{\prime}$ meets every boundary orbit of $D_{a}$. That completes the proof.

Proposition 2.13 combines with Lemmas 3.10 and 3.11 to give us

Proposition 3.12. Let $D$ be an open orbit of $S U(p, q)$ on the Grassmann manifold $\operatorname{Gr}(p, q)$. Then the Barlet-Koziarz intersection method shows that $M_{D}$ is Stein.

Proof. We have all of (2.11) except (2.11)(ii). But (2.11)(ii) was only used to show that $X_{a}^{\prime}$ meets every cycle $Y \in M_{D}$, and we proved that directly as Lemma 3.10. Thus, essentially as in Proposition 2.13, we conclude that $M_{D}$ is Stein. 


\section{Cayley transforms and boundary structure}

In this Section we recall the explicit real group orbit structure of flag manifolds that are the compact duals to bounded symmetric domains. That uses the Cayley transform methods of [5], [6] and [7], as described in [8], and extends (3.1), (3.2), (3.3) and (3.4) to the more general setting of hermitian symmetric spaces.

Let $B=G_{0} / K_{0}$ be an irreducible hermitian symmetric space of noncompact type, in other words an irreducible bounded symmetric domain. The real Lie algebra decomposes as usual into $\mathfrak{g}_{0}=\mathfrak{k}_{0}+\mathfrak{m}_{0}$ where $\mathfrak{m}_{0}$ represents the real tangent space, $\left[\mathfrak{k}_{0}, \mathfrak{m}_{0}\right]=\mathfrak{m}_{0},\left[\mathfrak{m}_{0}, \mathfrak{m}_{0}\right]=\mathfrak{k}_{0}$, and $\mathfrak{k}_{0}$ acts irreducibly (but not absolutely irreducibly) on $\mathfrak{m}_{0}$. The complexified Lie algebra decomposes as

(4.1) $\mathfrak{g}=\mathfrak{m}^{+}+\mathfrak{k}+\mathfrak{m}^{-}$where $\left[\mathfrak{k}, \mathfrak{m}^{ \pm}\right]=\mathfrak{m}^{ \pm},\left[\mathfrak{m}^{ \pm}, \mathfrak{m}^{ \pm}\right]=0$, and $\left[\mathfrak{m}^{-}, \mathfrak{m}^{+}\right]=\mathfrak{k}$.

The holomorphic tangent space of $B$ is represented by $\mathfrak{m}^{+}$, and $\mathfrak{m}^{-}=\overline{\mathfrak{m}^{+}}$ represents the antiholomorphic tangent space. The algebra $\mathfrak{k}$ acts irreducibly on each of $\mathfrak{m}^{ \pm}$. From (4.1) we have

$$
\begin{aligned}
\mathfrak{q}=\mathfrak{k}+\mathfrak{m}^{-} & \text {: parabolic subalgebra of } \mathfrak{g} \\
& \text { with nilradical } \mathfrak{m}^{-}, \text {reductive part } \mathfrak{k} .
\end{aligned}
$$

We may assume that $G_{0}$ is contained as a real form in the connected simply connected complex simple Lie group $G$ with Lie algebra $\mathfrak{g}$. Let $Q \subset G$ denote the parabolic subgroup with Lie algebra $\mathfrak{q}$ as in (4.2). Denote

$$
X=G / Q \text { complex flag manifold and } x_{0}=1 Q \in X \text { base point. }
$$

The Borel embedding of $B$ is

$$
B \cong G_{0}\left(x_{0}\right), \text { open } G_{0} \text {-orbit on } X .
$$

Finally, we note that one can identify $X$ with the compact dual symmetric space $G_{u} / K_{0}$ of $B=G_{0} / K_{0}$. For that, $G_{u}$ is the compact real form of $G$ with Lie algebra $\mathfrak{g}_{u}=\mathfrak{k}_{0}+\sqrt{-1} \mathfrak{m}_{0}$, and $G_{u}$ acts transitively on $X$ with isotropy $K_{0}$ at $x_{0}$.

Choose a Cartan subalgebra $\mathfrak{t}_{0} \subset \mathfrak{k}_{0}$; it also is a Cartan subalgebra of $\mathfrak{g}_{0}$. Fix any positive root system $\Delta^{+}(\mathfrak{k}, \mathfrak{t})$. Extend it to a positive root system $\Delta^{+}=$ $\Delta^{+}(\mathfrak{g}, \mathfrak{t})$ by requiring that $\mathfrak{m}^{+}$be a sum of positive root spaces, thus that $\mathfrak{m}^{-}$be a sum of negative root spaces. Roots $\alpha, \beta$ are strongly orthogonal, written $\alpha \perp \beta$, if neither of $\alpha \pm \beta$ is a root. Consider the "cascade construction"

$$
\Psi=\left\{\psi_{1}, \ldots, \psi_{\ell}\right\} \text {, maximal set constructed by: }
$$

$\psi_{1}$ is the maximal root in $\Delta^{+}$(always noncompact),

$$
\psi_{i+1} \text { is a maximal root in } \Delta\left(\mathfrak{m}^{+}, \mathfrak{t}\right) \text { with } \psi_{i+1} \perp\left\{\psi_{1}, \ldots, \psi_{i}\right\} \text {. }
$$

Any two different roots of $\Psi$ are strongly orthogonal, so $\Psi$ is a maximal set of strongly orthogonal noncompact positive roots. Any maximal set of strongly 
orthogonal noncompact positive roots is. $K_{0}$-conjugate to the one constructed in (4.5).

If $\alpha \in \Delta$ then $h_{\alpha} \in \sqrt{-1} \mathfrak{t}_{0}$ is defined by $\frac{2 \alpha(h)}{\langle\alpha, \alpha\rangle}=\left\langle h_{\alpha}, h\right\rangle$ for all $h \in \mathfrak{t}$. One can choose root vectors $e_{\alpha} \in \mathfrak{g}^{\alpha}$, normalized by $\left[e_{\alpha}, e_{-\alpha}\right]=h_{\alpha}$, such that the

$$
x_{\alpha, 0}=e_{\alpha}+e_{-\alpha} \text { and } y_{\alpha, 0}=\sqrt{-1}\left(e_{\alpha}-e_{-\alpha}\right) \text { for } \alpha \in \Delta^{+}(\mathfrak{m}, \mathfrak{t})
$$

form a real basis of $\mathfrak{m}_{0}$. Here $\left[x_{\alpha, 0}, y_{\alpha, 0}\right]=-2 \sqrt{-1} h_{\alpha}$. The almost complex structure $J$ is given by a central element $z \in \mathfrak{k}_{0}$; in the basis (4.6) it is

$$
J x_{\alpha, 0}=\left[z, x_{\alpha, 0}\right]=y_{\alpha, 0} \text { and } J y_{\alpha, 0}=\left[z, y_{\alpha, 0}\right]=-x_{\alpha, 0} .
$$

Similarly (or consequently) $\mathfrak{m}_{u}=\sqrt{-1} \mathfrak{m}_{0}$ has a real basis consisting of the

$$
x_{\alpha}=\sqrt{-1} x_{\alpha, 0}=\sqrt{-1}\left(e_{\alpha}+e_{-\alpha}\right) \text { and } y_{\alpha}=\sqrt{-1} y_{\alpha, 0}=-\left(e_{\alpha}-e_{-\alpha}\right)
$$

for $\alpha \in \Delta^{+}(\mathfrak{m}, \mathfrak{t})$. From maximal strong orthogonality of $\Psi$ we have maximal commutative subspaces

$$
\mathfrak{a}_{0}=\sum_{\psi \in \Psi} x_{\psi, 0} \mathbb{R} \subset \mathfrak{m}_{0} \text { and } \mathfrak{a}_{u}=\sum_{\psi \in \Psi} x_{\psi} \mathbb{R} \subset \mathfrak{m}_{u}
$$

Given $\psi \in \Psi$ we have the 3-dimensional simple algebra

$$
\mathfrak{g}[\psi]=\mathfrak{g}^{\psi}+\mathfrak{g}^{-\psi}+h_{\psi} \mathbb{C} \cong \mathfrak{s l}(2 ; \mathbb{C}) \text { in } \mathfrak{g},
$$

its noncompact real form

$$
\mathfrak{g}_{0}[\psi]=\mathfrak{g}[\psi] \cap \mathfrak{g}_{0}=\operatorname{Span}\left\{x_{\psi, 0}, y_{\psi, 0}, \sqrt{-1} h_{\psi}\right\} \cong \mathfrak{s l}(2 ; \mathbb{R}),
$$

and its compact real form

$$
\mathfrak{g}_{u}[\psi]=\mathfrak{g}[\psi] \cap \mathfrak{g}_{u}=\operatorname{Span}\left\{x_{\psi}, y_{\psi}, \sqrt{-1} h_{\psi}\right\} \cong \mathfrak{s u}(2),
$$

They define 3-dimensional simple subgroups

$$
G[\psi] \subset G \text { for } \mathfrak{g}[\psi], \quad G_{0}[\psi] \subset G_{0} \text { for } \mathfrak{g}_{0}[\psi], \quad G_{u}[\psi] \subset G_{u} \text { for } \mathfrak{g}_{u}[\psi] .
$$

Strong orthogonality of $\Psi$ says that $\left[\mathfrak{g}[\psi], \mathfrak{g}\left[\psi^{\prime}\right]\right]=0$ for $\psi, \psi^{\prime} \in \Psi$ with $\psi \neq \psi^{\prime}$. If $\Gamma \subset \Psi$ it follows that the

$$
\mathfrak{g}[\Gamma]=\sum_{\psi \in \Gamma} \mathfrak{g}[\psi] \subset \mathfrak{g}, \quad \mathfrak{g}_{0}[\Gamma]=\sum_{\psi \in \Gamma} \mathfrak{g}_{0}[\psi] \subset \mathfrak{g}_{0}, \quad \mathfrak{g}_{u}[\Gamma]=\sum_{\psi \in \Gamma} \mathfrak{g}_{u}[\psi] \subset \mathfrak{g}_{u}
$$

are well defined subalgebras that are Lie algebra direct sums. The corresponding groups

$$
\begin{aligned}
G[\Gamma]=\prod_{\psi \in \Gamma} G[\psi] \subset G, \quad G_{0}[\Gamma]=\prod_{\psi \in \Gamma} G_{0}[\psi] \subset G_{0} & \\
& G_{u}[\Gamma]=\prod_{\psi \in \Gamma} G_{u}[\psi] \subset G_{u}
\end{aligned}
$$


are locally direct products. Their orbits at the base point are

$$
\begin{aligned}
& \text { polydisk : } B[\Gamma]=G_{0}[\Gamma]\left(x_{0}\right), \text { product of }|\Gamma| \text { unit disks, and } \\
& \text { polysphere }: X[\Gamma]=G[\Gamma]\left(x_{0}\right)=G_{u}[\Gamma]\left(x_{0}\right), \text { product of }|\Gamma|
\end{aligned}
$$

Riemann spheres.

The (partial) Cayley transforms follow the classical model for the Riemann spheres of (4.16). If $\Gamma \subset \Psi$ we define the partial Cayley transform

$$
c_{\Gamma}=\prod_{\psi \in \Gamma} c_{\psi} \in G_{u}[\Psi] \subset G_{u} \quad \text { where } \quad c_{\psi}=\exp \left(\frac{\pi}{4} y_{\psi}\right) \in G_{u}[\psi]
$$

Now define points and orbits in $X$ by

$$
\text { if } \Gamma, \Sigma \subset \Psi \text { are disjoint, then } x_{\Gamma, \Sigma}=c_{\Gamma} c_{\Sigma}^{2} x_{0} \text { and } D_{\Gamma, \Sigma}=G_{0}\left(x_{\Gamma, \Sigma}\right) \text {. }
$$

We reformulate the Orbit Structure Theorem ([7, Theorem 10.6], or see [8, §7]):

Theorem 4.19. Assume that $B=G_{0} / K_{0}$ is irreducible, so $G$ is simple. The $G_{0}$-orbits on $X$ are just the $D_{\Gamma, \Sigma}$ where $\Gamma$ and $\Sigma$ are disjoint subsets of $\Psi$. An orbit $D_{\Gamma^{\prime}, \Sigma^{\prime}}$ is in the closure of $D_{\Gamma, \Sigma}$ (where $\Gamma \cap \Sigma=\emptyset=\Gamma^{\prime} \cap \Sigma^{\prime}$ ) if and only if the cardinalities $\left|\Sigma^{\prime}\right| \leqq|\Sigma|$ and $|\Sigma \cup \Gamma| \leqq\left|\Sigma^{\prime} \cup \Gamma^{\prime}\right|$. In particular

(i) $D_{\Gamma^{\prime}, \Sigma^{\prime}}=D_{\Gamma, \Sigma}$ if and only if both $|\Gamma|=\left|\Gamma^{\prime}\right|$ and $|\Sigma|=\left|\Sigma^{\prime}\right|$;

(ii) the number of $G_{0}$-orbits on $X$ is $(\ell+1)(\ell+2)$ where $\ell=|\Psi|$;

(iii) $D_{\Gamma, \Sigma}$ is open in $X$ if and only if $\Gamma$ is empty, so the open $G_{0}$-orbits are the $\ell+1$ orbits $\left\{D_{0}, \ldots, D_{\ell}\right\}$ where $D_{|\Sigma|}=D_{\emptyset, \Sigma}$ for $0 \leqq|\Sigma| \leqq \ell$;

(iv) bd $\left(D_{i}\right)$ is the union of the $D_{\Gamma^{\prime}, \Sigma^{\prime}} \neq D_{i}$ with $\left|\Sigma^{\prime}\right| \leqq i \leqq\left|\Sigma^{\prime} \cup \Gamma^{\prime}\right|$; and

(v) $D_{\Psi, \emptyset}$ is the unique closed $G_{0}$-orbit on $X$; it is in the closure of every orbit and is the Bergman-Shilov boundary of $B=D_{0}$.

Theorem 4.19 gives us the boundary information that we will need in order to extend Proposition 3.12 from Grassmann manifolds with $G_{0}=S U(p, q)$, to all hermitian symmetric spaces. The boundary orbit information (3.1), (3.2), (3.3) and (3.4), for the case where $G_{0}=S U(p, q)$, can of course, be extracted from Theorem 4.19 .

\section{Construction and analysis of the transverse variety}

Retain the setup and notation of Section 4. In this section we will construct subvarieties $X_{i}^{\prime} \subset X$ for $0 \leqq i \leqq \ell$ such that $X_{i}^{\prime}$ satisfies (2.11) for the open orbit $D_{i}$. For this we have to define certain subspaces of $\mathfrak{g}_{0}$ using the partial Cayley transforms (4.17). 
Fix a subset $\Sigma \subset \Psi$. It is a fact [6] that $\operatorname{Ad}\left(c_{\Sigma}^{4}\right)$ has square 1 as an automorphism of $\mathfrak{g}$, and that it preserves both $\mathfrak{m}$ and $\mathfrak{g}_{0}$. Define

$$
\begin{aligned}
& \mathfrak{g}^{\Sigma}=\left\{\xi \in \mathfrak{g} \mid \operatorname{Ad}\left(c_{\Sigma}^{4}\right) \xi=\xi\right\} \quad \text { and } \quad \mathfrak{g}_{0}^{\Sigma}=\mathfrak{g}^{\Sigma} \cap \mathfrak{g}_{0}, \\
& \mathfrak{k}^{\Sigma}=\left\{\xi \in \mathfrak{k} \mid \operatorname{Ad}\left(c_{\Sigma}^{4}\right) \xi=\xi\right\} \quad \text { and } \quad \mathfrak{k}_{0}^{\Sigma}=\mathfrak{k}^{\Sigma} \cap \mathfrak{g}_{0}, \\
& \mathfrak{m}^{\Sigma}=\left\{\xi \in \mathfrak{m} \mid \operatorname{Ad}\left(c_{\Sigma}^{4}\right) \xi=\xi\right\} \quad \text { and } \quad \mathfrak{m}_{0}^{\Sigma}=\mathfrak{m}^{\Sigma} \cap \mathfrak{g}_{0}, \\
& \mathfrak{r}^{\Sigma}=\left\{\xi \in \mathfrak{m} \mid \operatorname{Ad}\left(c_{\Sigma}^{4}\right) \xi=-\xi\right\} \quad \text { and } \quad \mathfrak{r}_{0}^{\Sigma}=\mathfrak{r}^{\Sigma} \cap \mathfrak{g}_{0} .
\end{aligned}
$$

So $\mathfrak{g}^{\Sigma}=\mathfrak{k}^{\Sigma}+\mathfrak{m}^{\Sigma}$ and $\mathfrak{g}_{0}^{\Sigma}=\mathfrak{k}_{0}^{\Sigma}+\mathfrak{m}_{0}^{\Sigma}$.

The case of [7, Lemma 11.6] (or see [8, Lemma 9.10]), where $\Gamma$ is empty, says

Lemma 5.2. Define $f: K_{0} \times \mathfrak{m}_{0}^{\Sigma} \times \mathfrak{r}_{0}^{\Sigma} \rightarrow G_{0}$ by $f(k, \xi, \eta)=k \exp (\xi) \exp (\eta)$. Then $f$ is a real analytic diffeomorphism of $K_{0} \times \mathfrak{m}_{0}^{\Sigma} \times \mathfrak{r}_{0}^{\Sigma}$ onto $G_{0}$.

Our use of Lemma 5.2 will require a refinement of the notation (5.1). If $\Gamma \subset \Phi$ we note that the centralizer of $\mathfrak{g}[\Psi \backslash \Gamma]$ has form (subspace of $\mathfrak{t}$ ) $+\sum_{\alpha \perp \Psi \backslash \Gamma} \mathfrak{g}^{\alpha}$. It is a reductive algebra with semisimple part

$$
\mathfrak{g}_{\Gamma}=\mathfrak{k}_{\Gamma}+\mathfrak{m}_{\Gamma}: \text { derived algebra of } \mathfrak{t}+\sum_{\alpha \perp \Psi \backslash \Gamma} \mathfrak{g}^{\alpha} .
$$

which in turn has real forms

$$
\mathfrak{g}_{\Gamma, 0}=\mathfrak{g}_{0} \cap \mathfrak{g}_{\Gamma}=\mathfrak{k}_{\Gamma, 0}+\mathfrak{m}_{\Gamma, 0} \quad \text { and } \quad \mathfrak{g}_{\Gamma, u}=\mathfrak{g}_{u} \cap \mathfrak{g}_{\Gamma}=\mathfrak{k}_{\Gamma, u}+\mathfrak{m}_{\Gamma, u} .
$$

Of course we have the corresponding analytic groups

$$
G_{\Gamma} \subset G, \quad G_{\Gamma, 0} \subset G_{0} \quad \text { and } \quad G_{\Gamma, u} \subset G_{u}
$$

and their orbits

$$
X_{\Gamma}=G_{\Gamma}\left(x_{0}\right)=G_{\Gamma, u}\left(x_{0}\right) \subset X \quad \text { and } \quad B_{\Gamma}=G_{\Gamma, 0}\left(x_{0}\right)=B \cap X_{\Gamma} .
$$

Note that $\mathfrak{g}_{\Gamma}$ has $\Gamma$ as its maximal set of strongly orthogonal noncompact roots. In effect, these groups and spaces repeat the situation of $G_{0}$ and $X$ with $\Psi$ reduced to $\Gamma$. Passage from $G_{0}$ to $G_{\Gamma, 0}$ was exemplified in $\S 3$ by passage from $S U(p, q)$ to $S U(p-a, q-a)$ with $a=|\Psi \backslash \Gamma|$.

We combine the idea of (5.1) with that of (5.3) and (5.4). If $\Sigma \subset \Phi \subset \Psi$, define

$$
\begin{aligned}
& \mathfrak{g}_{\Phi}^{\Sigma}=\left\{\xi \in \mathfrak{g}_{\Phi} \mid \operatorname{Ad}\left(c_{\Sigma}^{4}\right) \xi=\xi\right\}=\mathfrak{g}_{\Phi} \cap \mathfrak{g}^{\Sigma} \text { and } \mathfrak{g}_{\Phi, 0}^{\Sigma}=\mathfrak{g}_{\Phi}^{\Sigma} \cap \mathfrak{g}_{0}, \\
& \mathfrak{k}_{\Phi}^{\Sigma}=\left\{\xi \in \mathfrak{k}_{\Phi} \mid \operatorname{Ad}\left(c_{\Sigma}^{4}\right) \xi=\xi\right\}=\mathfrak{k}_{\Phi} \cap \mathfrak{k}^{\Sigma} \text { and } \mathfrak{k}_{\Phi, 0}^{\Sigma}=\mathfrak{k}_{\Phi}^{\Sigma} \cap \mathfrak{k}_{0}, \\
& \mathfrak{m}_{\Phi}^{\Sigma}=\left\{\xi \in \mathfrak{m}_{\Phi} \mid \operatorname{Ad}\left(c_{\Sigma}^{4}\right) \xi=\xi\right\}=\mathfrak{m}_{\Phi} \cap \mathfrak{m}^{\Sigma} \text { and } \mathfrak{m}_{\Phi, 0}^{\Sigma}=\mathfrak{m}_{\Phi}^{\Sigma} \cap \mathfrak{m}_{0} .
\end{aligned}
$$

Then of course $\mathfrak{g}_{\Phi}^{\Sigma}=\mathfrak{k}_{\Phi}^{\Sigma}+\mathfrak{m}_{\Phi}^{\Sigma}$. We have real forms

$$
\mathfrak{g}_{\Phi, 0}^{\Sigma}=\mathfrak{g}_{0} \cap \mathfrak{g}_{\Phi}^{\Sigma}=\mathfrak{k}_{\Phi, 0}^{\Sigma}+\mathfrak{m}_{\Phi, 0}^{\Sigma} \quad \text { and } \quad \mathfrak{g}_{\Phi, u}^{\Sigma}=\mathfrak{g}_{u} \cap \mathfrak{g}_{\Phi}^{\Sigma}=\mathfrak{k}_{\Phi, u}^{\Sigma}+\mathfrak{m}_{\Phi, u}^{\Sigma}
$$

and analytic groups

$$
G_{\Phi}^{\Sigma} \subset G, \quad G_{\Phi, 0}^{\Sigma} \subset G_{0} \quad \text { and } \quad G_{\Phi, u}^{\Sigma} \subset G_{u}
$$


and their orbits

$$
X_{\Phi}^{\Sigma}=G_{\Phi}^{\Sigma}\left(x_{0}\right)=G_{\Phi, u}^{\Sigma}\left(x_{0}\right) \subset X \quad \text { and } \quad B_{\Phi}^{\Sigma}=G_{\Phi, 0}^{\Sigma}\left(x_{0}\right)=B \cap X_{\Phi}^{\Sigma} .
$$

Here notice $G_{\Phi}^{\emptyset}=G_{\Phi}, \quad G_{\Phi, 0}^{\emptyset}=G_{\Phi, 0}, G_{\Phi, u}^{\emptyset}=G_{\Phi, u}, \quad X_{\Phi}^{\emptyset}=X_{\Phi}$, and $B_{\Phi}^{\emptyset}=$ $B_{\Phi}$.

Lemma 5.11. If $\Sigma \subset \Psi$ then

$$
\begin{aligned}
& G^{\Sigma}\left(x_{0}\right)=X^{\Sigma} \cong\left(X_{\Psi \backslash \Sigma} \times X_{\Sigma}^{\Sigma}\right)=\left(G_{\Psi \backslash \Sigma}\left(x_{0}\right) \times G_{\Sigma}^{\Sigma}\left(x_{0}\right)\right) \text { and } \\
& G_{0}^{\Sigma}\left(x_{0}\right)=B^{\Sigma} \cong\left(B_{\Psi \backslash \Sigma} \times B_{\Sigma}^{\Sigma}\right)=\left(G_{\Psi \backslash \Sigma, 0}\left(x_{0}\right) \times G_{\Sigma, 0}^{\Sigma}\left(x_{0}\right)\right) .
\end{aligned}
$$

Proof. This is essentially [7, Theorem 11.8 (1d)] with $\Gamma=\emptyset$. It is based on the argument in the proof (see $\left[7\right.$, p. 1215]) that $\mathfrak{g}^{\Sigma}=\mathfrak{g}_{\Psi \backslash \Sigma} \oplus \mathfrak{g}_{\Sigma}^{\Sigma} \oplus \mathfrak{l}_{\emptyset, \Sigma}$ where $\mathfrak{l}_{\emptyset, \Sigma} \subset \mathfrak{k}$.

Lemma 5.12. If $\Sigma \subset \Psi$ then

$$
G^{\Sigma}\left(x_{\emptyset, \Sigma}\right)=c_{\Sigma}^{2} G^{\Sigma}\left(x_{0}\right)=c_{\Sigma}^{2} X^{\Sigma} \text { and } G_{0}^{\Sigma}\left(x_{\emptyset, \Sigma}\right)=c_{\Sigma}^{2} G_{0}^{\Sigma}\left(x_{0}\right)=c_{\Sigma}^{2} B^{\Sigma} .
$$

Proof. This also is implicit in [7, Theorem $11.8(1 \mathrm{~d})]$ with $\Gamma=\emptyset$. By construction, $\operatorname{Ad}\left(c_{\Sigma}^{2}\right)$ normalizes $\mathfrak{g}^{\Sigma}$. Thus $G^{\Sigma}\left(x_{\emptyset, \Sigma}\right)=G^{\Sigma}\left(c_{\Sigma}^{2} x_{0}\right)=c_{\Sigma}^{2} G^{\Sigma}\left(x_{0}\right)=$ $c_{\Sigma}^{2} X^{\Sigma}$.

$\operatorname{Ad}\left(c_{\Sigma}^{2}\right)_{\mathfrak{g}^{\Sigma}}$ has square 1 and commutes with both the Cartan involution $\theta$ and the complex conjugation $\xi \mapsto \bar{\xi}$ of $\mathfrak{g}$ over $\mathfrak{g}_{0}$. So $\operatorname{Ad}\left(c_{\Sigma}^{2}\right)$ preserves both $\mathfrak{g}_{0}^{\Sigma}$ and its decomposition $\mathfrak{g}_{0}^{\Sigma}=\mathfrak{k}_{0}^{\Sigma}+\mathfrak{m}_{0}^{\Sigma}$. Now $\operatorname{Ad}\left(c_{\Sigma}^{2}\right)$ normalizes $G_{0}^{\Sigma}$ and its maximal compact subgroup $K_{0}^{\Sigma}$. Thus $G_{0}^{\Sigma}\left(x_{\emptyset, \Sigma}\right)=G_{0}^{\Sigma}\left(c_{\Sigma}^{2} x_{0}\right)=c_{\Sigma}^{2} G_{0}^{\Sigma}\left(x_{0}\right)=c_{\Sigma}^{2} B^{\Sigma}$.

Fix the open orbit $D=D_{|\Sigma|}$ and let $Y_{0}$ denote its base cycle, the maximal compact subvariety $K\left(x_{\emptyset, \Sigma}\right)=K_{0}\left(x_{\emptyset, \Sigma}\right)$. Now use Lemma 5.2 to define a map

$$
\pi: D \rightarrow Y_{0} \text { by } \pi(k \exp (\xi) \exp (\eta))\left(x_{\emptyset, \Sigma}\right)=k\left(x_{\emptyset, \Sigma}\right) .
$$

The maps $\beta_{k}$ of the Orbit Fibration Theorem ([7, Theorem 11.8], or see [8, $\S 9])$ are well defined $C^{\omega}$ fibrations ${ }^{1}$. When $\Gamma=\emptyset$ the Orbit Fibration Theorem implies

Lemma 5.14. The map $\pi$ of (5.13) is a well defined $K_{0}$-equivariant real analytic fibration of $D$ over $Y_{0}$. The fiber over $x_{\emptyset, \Sigma}$ is $c_{\Sigma}^{2} B^{\Sigma}=c_{\Sigma}^{2}\left(B_{\Psi \backslash \Sigma} \times B_{\Sigma}^{\Sigma}\right)$.

Proof. The fact that $\pi$ is a real analytic fibration, is contained in [7] and [8] for $\Gamma=\emptyset$. It is also shown there that the fiber $\pi^{-1}\left(x_{\emptyset, \Sigma}\right)=G_{0}^{\Sigma}\left(x_{\emptyset, \Sigma}\right)$. The precise description of the fiber, asserted here, now follows from Lemmas 5.11 and 5.12 .

Lemma 5.15. If $\Sigma \subset \Psi$ then $X^{\Sigma}=c_{\Sigma}^{2} X^{\Sigma}=c_{\Sigma}^{2}\left(X_{\Psi \backslash \Sigma} \times X_{\Sigma}^{\Sigma}\right)$, and it satisfies (2.11) for the open $G_{0}$-orbit $D=D_{|\Sigma|}$ on $X$.

\footnotetext{
${ }^{1}$ There may be a problem with holomorphicity of the $\beta_{k}$ but that is not of concern to us here.
} 
Proof. Use Lemma 5.14 to see that $\operatorname{dim} c_{\Sigma}^{2} X^{\Sigma}=\operatorname{dim} c_{\Sigma}^{2} B^{\Sigma}=\operatorname{dim} \pi^{-1}\left(x_{\emptyset, \Sigma}\right)=$ $\operatorname{dim} D-\operatorname{dim} Y_{0}$, so the codimension of $\operatorname{dim} c_{\Sigma}^{2} X^{\Sigma}$ in $X$ is $\operatorname{dim} Y_{0}$, as required for $(2.11)(\mathrm{i})$.

The base point $x_{\emptyset, \Sigma}$ of $D=D_{|\Sigma|}$ is contained in both $X^{\Sigma}$ and in $Y_{0}$, and Lemma 5.14 ensures that the intersection at that point is transversal. Thus we have (2.11)(ii).

Now we must show that $c_{\Sigma}^{2} X^{\Sigma} \cap D=c_{\Sigma}^{2} B^{\Sigma}$, which is a bounded symmetric domain, thus Stein. This is a consequence of [11, Theorem 3.8] as follows. Our $X$ is both the hermitian symmetric space $X$ of [11] and the complex flag manifold $Z$ of [11]. Our $x_{0}$ is the base point for both. Now the $G_{0}$-orbits on $X$ are the $D_{\Gamma, \Sigma}$ as described in Theorem 4.19. Our $\operatorname{Ad}\left(c_{\Sigma}^{4}\right)$ is the involutive automorphism $\sigma$ of [11]. The Cartan involution $\theta$ satisfies $\theta\left(y_{\psi}\right)=-y_{\psi}$, so $\theta\left(c_{\psi}\right)=c_{\psi}^{-1}$ and this $\theta\left(c_{\Sigma}\right)=c_{\Sigma}^{-1}$, so $\theta \operatorname{Ad}\left(c_{\Sigma}^{4}\right)=\operatorname{Ad}\left(c_{\Sigma}^{-4}\right) \theta=\operatorname{Ad}\left(c_{\Sigma}^{4}\right) \theta$; so $\operatorname{Ad}\left(c_{\Sigma}^{4}\right)$ commutes with $\theta$ as required. Now our $G^{\Sigma}$ and its real form $G_{0}^{\Sigma}$, fixed point groups in $G$ and $G_{0}$ of $\operatorname{Ad}\left(c_{\Sigma}^{4}\right)$, are the fixed point groups $M$ and $M_{0}$ of $\sigma$ in [11]. Our $X^{\Sigma}=G^{\Sigma}\left(x_{0}\right)$ is the space $F=M(z)$ in [11]. Note that $\mathfrak{g}[\Psi] \subset \mathfrak{g}^{\Sigma}$, so our $\Psi$ is the $\Psi^{\mathfrak{m}}$ of [11]. Thus [11, Theorem 3.8], especially the last sentence of the theorem, which is hidden at the top of page 400 there, gives the following. As $\Phi$ and $\Gamma$ range over disjoint pairs of subsets of $\Psi$,

$$
G_{0}^{\Sigma}\left(x_{\Gamma, \Phi}\right) \mapsto G_{0}\left(x_{\Gamma, \Phi}\right)=D_{\Gamma, \Phi} \text { is a one-one map }
$$

from the set of $G_{0}^{\Sigma}$-orbits on $X^{\Sigma}$ onto the set of $G_{0}$-orbits on $X$.

Conclusion: $G_{0}^{\Sigma}\left(x_{\emptyset, \Sigma}\right)=c_{\Sigma}^{2} B^{\Sigma}$ is the only $G_{0}^{\Sigma}$-orbit in $c_{\Sigma}^{2} X^{\Sigma} \cap D$. So $c_{\Sigma}^{2} X^{\Sigma} \cap D$ $=c_{\Sigma}^{2} B^{\Sigma}$ is a bounded symmetric domain, thus Stein, as required for (2.11)(iii).

According to Theorem 4.19 the $G_{0}$-orbits in the boundary of $D=D_{\Sigma}$ are those $D_{\Gamma^{\prime}, \Sigma^{\prime}} \neq D$ with $\Sigma^{\prime} \subset \Sigma \subset\left(\Sigma^{\prime} \cup \Gamma^{\prime}\right)$. But as noted above, $\mathfrak{g}[\Psi] \subset \mathfrak{g}^{\Sigma}$, so every $x_{\Gamma^{\prime}, \Sigma^{\prime}} \in X^{\Sigma}=c_{\Sigma}^{2} X^{\Sigma}$. Thus $X^{\Sigma}$ meets every $G_{0}$-orbit on the boundary of $D$. Now we have (2.11)(iv). This completes the verification of (2.11), and thus the proof of Lemma 5.15 .

Proposition 2.13 combines with Lemma 5.15 to give us

Proposition 5.17. Let $D$ be an open $G_{0}$-orbit the hermitian symmetric flag manifold $X$. Then the Barlet-Koziarz intersection method shows that $M_{D}$ is Stein.

\section{References}

[1] D. Barlet, Convexité de l'espace des cycles, Bull. Soc. Math. France 106 (1978), 373-397.

[2] D. Barlet and V. Koziarz, Fonctions holomorphes sur l'espace des cycles: la méthode d'intersection, Math. Res. Letters 7 (2000), 537-549.

[3] A. T. Huckleberry and A. Simon, On cycle spaces of flag domains of $S L_{n}(\mathbb{R})$, to appear.

[4] A. T. Huckleberry and J. A. Wolf, Flag duality, Ann. Global Anal. Geom., to appear.

[5] A. Korányi and J. A. Wolf, Realization of hermitian symmetric spaces as generalized mboxhalf-planes, Ann. of Math. 81 (1965), 265-288. 
[6] - Generalized Cayley transformations of bounded symmetric domains, Amer. J. Math. 87 (1965), 899-939.

[7] J. A. Wolf, The action of a real semisimple group on a complex flag manifold I: Orbit structure and holomorphic arc components, Bull. Amer. Math. Soc. 75 (1969), 1121-1237.

[8] - Fine structure of hermitian symmetric spaces, Symmetric spaces, Short Courses, Washington Univ., St. Louis, Mo., 1969-1970, 271-357. Pure and App. Math., 8 (1972).

[9] - The Stein condition for cycle spaces of open orbits on complex flag manifolds, Ann. of Math. 136 (1992), 541-555.

[10] - Exhaustion functions and cohomology vanishing theorems for open orbits on complex flag manifolds, Math. Res. Letters 2, (1995), 179-191.

[11] J. A. Wolf and R. Zierau, Cayley transforms and orbit structure in complex flag manifolds, Transform. Groups 2 (1997), 391-405.

[12] - Linear cycle spaces in flag domains, Math. Ann., to appear.

Institut für Mathematik, Ruhr-Universität Bochum, D-44780 Bochum, Germany

Department of Mathematics, Univ. of California, Berkeley, California 947203840, U.S.A.

E-mail address: jawolf@math.berkeley.edu 